\title{
Effect of Tin Codoping on Transport and Magnetic Properties of Chromium-doped Indium Oxide Films
}

\author{
Hyojin Kim ${ }^{1 *}$, Hyoun Soo Kim², Dojin Kim ${ }^{1}$, Young Eon $\mathrm{Ihm}^{1}$, Woong Kil $\mathrm{Choo}^{2}$, and Chanyong Hwang ${ }^{3}$ \\ ${ }^{1}$ Department of Nano Information Systems Engineering, School of Nano Science and Technology, \\ Chungnam National University, Daejeon 305-764, Korea \\ ${ }^{2}$ Department of Materials Science and Engineering, Korea Advanced Institute of Science and Technology, Daejeon 305-701, Korea \\ ${ }^{3}$ Advanced Industrial Technology Group, Division of Advanced Technology, Korea Research Institute of Standards and Science, \\ Daejeon 305-600, Korea
}

(Received 26 May 2008, Received in final form 7 September 2008, Accepted 9 September 2008)

\begin{abstract}
This study examined the effect of Sn co-doping on the transport and magnetic properties of Cr-doped $\mathrm{In}_{2} \mathrm{O}_{3}$ thin films grown on (100) silicon substrates by pulsed laser deposition. The experimental results showed that $\mathrm{Sn}$ co-doping enhances the magnetization and appearance of the anomalous Hall effect, and increases the carrier (electron) concentration. These results suggest that the conduction carrier plays an important role in enhancing the ferromagnetism of a laser-deposited $\mathrm{Cr}$-doped $\mathrm{In}_{2} \mathrm{O}_{3}$ film, which may have applications in transparent oxide semiconductor spin electronics devices.
\end{abstract}

Keywords : diluted magnetic semiconductors, indium tin oxide, ferromagnetism, magnetotransport

\section{Introduction}

Oxide based diluted magnetic semiconductors (DMS) have attracted considerable attention on account of their promising potential in spintronics. In particular, there have been some reports on the discovery of room temperature ferromagnetism in several oxide DMS [1-3]. Among these systems, $\mathrm{ZnO}$ - and $\mathrm{TiO}_{2}$-based DMS have been studied extensively as the most promising candidates with Curie temperatures well in excess of $300 \mathrm{~K}$, which are essential for realizing room temperature practical spintronic devices. However, the real origin of the ferromagnetism observed in these systems is not completely understood, and the possibility of an extrinsic origin of ferromagnetism, such as ferromagnetic clustering, cannot be excluded. The experimental results reported are not even reproducible, with different preparation methods yielding different magnetic phenomena. On the other hand, it has been suggested that crystal defects in oxide DMSs play a major role in inducing high temperature ferromagnetism [4-6].

Both theoretical and experimental studies suggest that

*Corresponding author: Tel: +82-42-821-6636

Fax: +82-42-822-3206, e-mail: hyojkim@cnu.ac.kr wide bandgap oxide semiconductors with a high carrier density are one of the most favorable host compounds for ferromagnetic DMS with a higher Curie temperature [2, 3]. Indium oxide, $\operatorname{In}_{2} \mathrm{O}_{3}$, is an excellent base material for a transparent conducting oxide with a tunable carrier density and high mobility as well as a wide band gap $>3.5$ $\mathrm{eV}$ [7]. The $n$-type conductivity in $\mathrm{In}_{2} \mathrm{O}_{3}$-based system is due to the electrons generated by oxygen deficiencies. Recently, $\mathrm{In}_{2} \mathrm{O}_{3}$-based DMSs have been found to exhibit both ferromagnetism and an anomalous Hall effect at room temperature $[6,8,9]$. In particular, $\mathrm{Cr}$-doped $\mathrm{In}_{2} \mathrm{O}_{3}$ is a unique system in that the magnetic doping and carrier concentration can be controlled independently to possibly show a high Curie temperature [6]. In this study, an attempt was made to vary the electron concentration by doping with $\mathrm{Sn}$, which acts as a donor in $\mathrm{In}_{2} \mathrm{O}_{3}$.

This paper reports the effect of $\mathrm{Sn}$ co-doping on the transport and magnetic properties of Cr-doped $\mathrm{In}_{2} \mathrm{O}_{3}$ in order to demonstrate the enhancement of carrier-mediated ferromagnetism in Cr-doped $\operatorname{In}_{2} \mathrm{O}_{3}$ by electron doping through Sn co-doping.

\section{Experiments}

All $\mathrm{Cr}+\mathrm{Sn}$ co-doped $\mathrm{In}_{2} \mathrm{O}_{3}\left[\left(\mathrm{In}_{0.95-x} \mathrm{Cr}_{0.05} \mathrm{Sn}_{x}\right)_{2} \mathrm{O}_{3}\right]$ films 
were prepared using a conventional pulsed laser deposition (PLD) method with a $\mathrm{KrF}$ excimer laser operating at $248 \mathrm{~nm}$ and an intensity of $5 \mathrm{~J} / \mathrm{cm}^{2}$. The ceramic targets were prepared from high-purity $\mathrm{In}_{2} \mathrm{O}_{3}, \mathrm{Cr}_{2} \mathrm{O}_{3}$ and $\mathrm{SnO}_{2}$ powders at certain molar ratios using standard solid-state reaction procedures. The mixed powders were cold pressed and sintered at $1300^{\circ} \mathrm{C}$ for $96 \mathrm{hr}$ in air. The substrates were $10 \times 10 \mathrm{~mm}^{2} \mathrm{SiO}_{2}(200 \mathrm{~nm}) / \mathrm{Si}(100)$ that maintained at $400^{\circ} \mathrm{C}$ at a fixed oxygen pressure of 100 mtorr during deposition. The film thickness ranged from 800-1000 nm. The film composition was determined by energy-dispersive X-ray (EDX) spectroscopy.

\section{Results and Discussion}

Conventional X-ray diffraction (XRD) using $\mathrm{Cu} \mathrm{K} \alpha$ radiation and secondary ion mass spectroscopy (SIMS) were used for structure analysis and phase identification of the synthesized films. Fig. 1 shows the XRD patterns of the $\mathrm{Cr}+\mathrm{Sn}$ co-doped $\mathrm{In}_{2} \mathrm{O}_{3}\left[\left(\mathrm{In}_{0.95-x} \mathrm{Cr}_{0.05} \mathrm{Sn}_{x}\right)_{2} \mathrm{O}_{3}\right]$ films with a fixed $\mathrm{Cr}$ concentration of 5 at.\% for $0 \leq x \leq 0.12$, where $x$ denotes the $\mathrm{Sn}$ concentration. The peak positions for $\mathrm{In}_{2} \mathrm{O}_{3}$ of a cubic bixbyite structure [10] are shown in the bottom panel of Fig. 1. All diffraction lines from the films were indexed assuming a bixbyite structure with no detectable secondary phases, indicating homogeneous singlephase growth without phase segregation. Furthermore, as shown in the insets of Fig. 1, the results of SIMS analysis for $x=0.05$ and 0.12 demonstrate a uniform distribution of $\mathrm{Cr}$ and $\mathrm{Sn}$ ions at different film depths, indicating no surface or interface segregation of the doped ions.

After structural characterization, the influence of Sn codoping on the transport properties of the Cr-doped $\operatorname{In}_{2} \mathrm{O}_{3}$

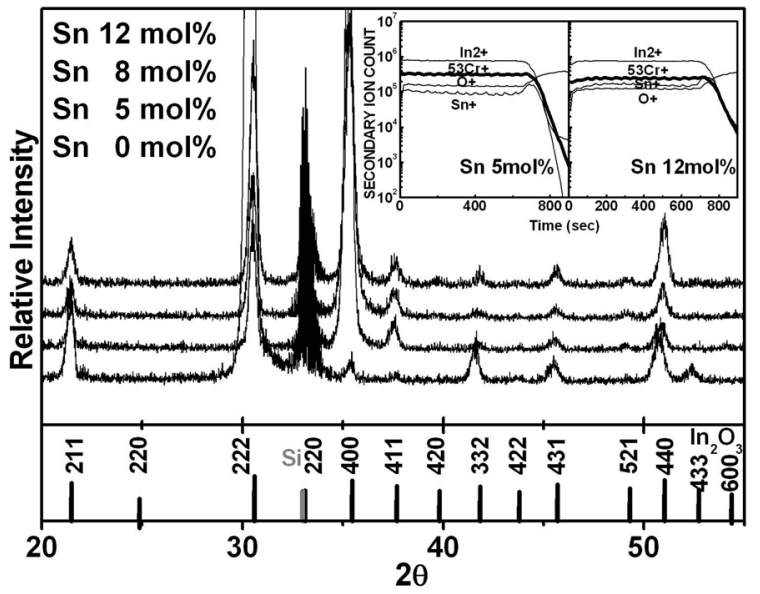

Fig. 1. X-ray diffraction patterns of the laser-deposited $\left(\operatorname{In}_{0.95-x^{-}}\right.$ $\left.\mathrm{Cr}_{0.05} \mathrm{Sn}_{x}\right)_{2} \mathrm{O}_{3}(0 \leq x \leq 0.12)$ films. The insets show the results of SIMS analysis for $x=0.05$ and 0.12 .

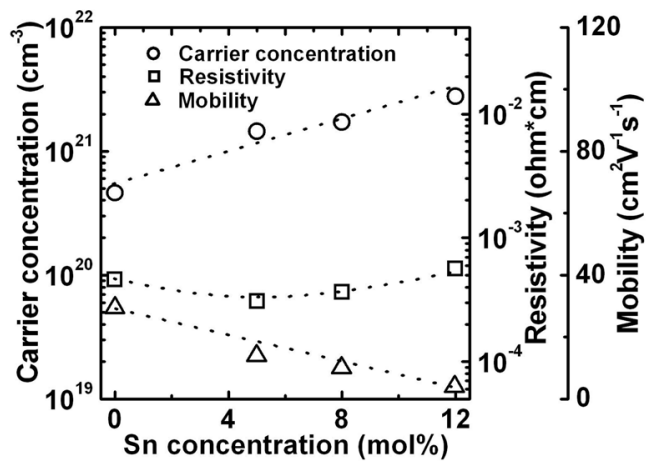

Fig. 2. Electron concentration, mobility and resistivity of the $\left(\mathrm{In}_{0.95-x} \mathrm{Cr}_{0.05} \mathrm{Sn}_{x}\right)_{2} \mathrm{O}_{3}(0 \leq x \leq 0.12)$ films, obtained from Hall effect measurements.

samples was examined by Hall measurements using the van der Pauw configuration at room temperature. Fig. 2 shows the change in carrier concentration, mobility and electrical resistivity as a function of the $\mathrm{Sn}$ concentration for the $\left(\mathrm{In}_{0.95-x} \mathrm{Cr}_{0.05} \mathrm{Sn}_{x}\right)_{2} \mathrm{O}_{3}$ films. All the samples showed $n$-type conductivity and the electron concentration increased from $4.61 \times 10^{20} \mathrm{~cm}^{-3}$ for $x=0$ to $2.78 \times 10^{21} \mathrm{~cm}^{-3}$ for $x$ $=0.12$ with Sn co-doping. This suggests that the Sn ion acts as a donor. On the other hand, there was a linear decrease in the mobility with increasing Sn codoping, which was attributed to an increase in alloy scattering caused by the presence of Sn ions. As a result, the films maintained a low resistivity of approximately $4 \times 10^{-4} \Omega$ $\mathrm{cm}$.

The effect of Sn co-doping to the magnetic properties
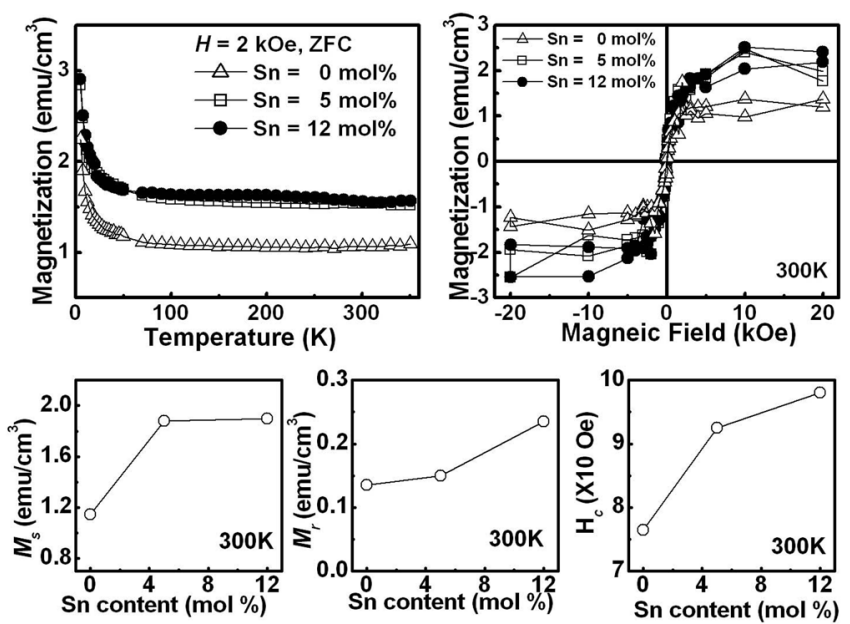

Fig. 3. (a) Magnetization versus temperature curves measured in a field of $2 \mathrm{kOe}$; (b) The magnetization versus magnetic field curves measured at $300 \mathrm{~K}$; (c) Saturation magnetization $\left(M_{\mathrm{S}}\right)$; (d) Remnant magnetization $\left(M_{\mathrm{r}}\right)$; (e) Coercive field $\left(H_{\mathrm{C}}\right)$ for the laser-deposited $\left(\operatorname{In}_{0.95-x} \mathrm{Cr}_{0.05} \mathrm{Sn}_{x}\right)_{2} \mathrm{O}_{3} \quad(x=0,0.05$ and 0.12) films. 
of Cr-doped $\mathrm{In}_{2} \mathrm{O}_{3}$ films was examined. Figure 3(a) shows the temperature dependence of the magnetization $(M-T$ curves) of the $\left(\operatorname{In}_{0.95-x} \mathrm{Cr}_{0.05} \mathrm{Sn}_{x}\right)_{2} \mathrm{O}_{3}$ films at three $\mathrm{Sn}$ concentrations $(x=0,0.05$ and 0.12$)$, which were measured using a superconducting quantum interference device (SQUID) magnetometer during warming from 5 to $350 \mathrm{~K}$ in an applied magnetic field $(H)$ of $2 \mathrm{kOe}$ parallel to the film surface. The diamagnetic contribution due to the substrate was subtracted. It should be noted that all three films have large magnetization values $>\sim 1 \mathrm{emu} / \mathrm{cm}^{3}$ and show similar magnetization at temperatures $>50 \mathrm{~K}$. This indicates the presence of a ferromagnetic ordering in the films. From the field dependence of the magnetization $(M-H$ curves) at $300 \mathrm{~K}$ for the three samples, shown in Fig. 3(b), clear ferromagnetic hysteresis loops were observed, showing further evidence for the ferromagnetism in the films. This is consistent with the discovery of ferromagnetism in Cr-doped $\operatorname{In}_{2} \mathrm{O}_{3}$ films with an electron concentration above $2 \times 10^{19} \mathrm{~cm}^{-3}$ [6], considering that the electron concentrations of the samples in the present study ranged from $0.4-3 \times 10^{21} \mathrm{~cm}^{-3}$. There was no noticeable difference in the $M-T$ curves between the zero-fieldcooled and field-cooled ones, indicating the absence of spin-glass-like behavior or magnetic cluster formation. The observed upturn in the $M-T$ curves at very low temperatures can be attributed to the paramagnetic $\mathrm{Cr}$ moments. Consequently, the characteristics of the $M-T$ curves can be well understood by the coexistence of the ferromagnetic and paramagnetic components, as observed in the Mn-doped $\mathrm{ZnO}$ [11], Co-doped $\mathrm{Cu}_{2} \mathrm{O}$ [12] and Mn-doped $\mathrm{GaN}$ [13].

Fig. 3(c), (d) and (e) show the changes in saturation magnetization $\left(M_{S}\right)$, remnant magnetization $\left(M_{r}\right)$ and the coercive field $\left(H_{C}\right)$ with $\mathrm{Sn}$ co-doping, respectively, which were obtained from the $M-H$ curves in Fig. 3(b). The increase in $M_{r}$ and $H_{C}$ as well as $M_{S}$ demonstrates the enhancement of ferromagnetism in Cr-doped $\mathrm{In}_{2} \mathrm{O}_{3}$ films co-doped with $\mathrm{Sn}$. This highlights the electron-dopingdriven enhancement of carrier-controlled ferromagnetism in Cr-doped $\mathrm{In}_{2} \mathrm{O}_{3}$ as reported by Philip et al. [6].

One of the important criteria for intrinsic ferromagnetism caused by spin-polarized carriers in magnetic semiconductors is the observation of an anomalous Hall effect (AHE). The Hall resistivity $\left(\rho_{x y}\right)$ in ferromagnets is customarily expressed as a sum of the ordinary and anomalous Hall term, $\rho_{x y}=R_{0} B+R_{S} M$, where $R_{0}$ is the ordinary Hall coefficient, $B$ is the magnetic induction and $R_{S}$ is the anomalous Hall coefficient [14]. The AHE, which is proportional to the magnetization $(M)$, is dominant at low magnetic fields and has a positive slope. Fig. 4 shows the magnetic field dependence of the Hall resistivity, $\rho_{x y}$, of

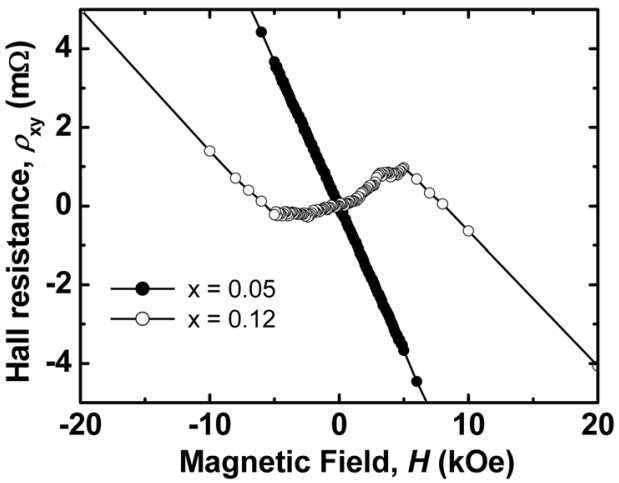

Fig. 4. Hall resistivity $\rho_{x y}$ as a function of the magnetic field for the laser-deposited $\left(\mathrm{In}_{0.95-x} \mathrm{Cr}_{0.05} \mathrm{Sn}_{x}\right)_{2} \mathrm{O}_{3}(x=0.05$ and 0.12) films.

the $\left(\mathrm{In}_{0.95-x} \mathrm{Cr}_{0.05} \mathrm{Sn}_{x}\right)_{2} \mathrm{O}_{3}$ films at $x=0.05$ and 0.12 at room temperature. For the $x=0.12$ film with an electron density of $2.78 \times 10^{21} \mathrm{~cm}^{-3}$, the Hall resistivity $\rho_{x y}$ increases linearly with increasing magnetic field at fields $<\sim 5 \mathrm{kOe}$, i.e. the AHE. At higher fields ( $>\sim 5 \mathrm{kOe}), \rho_{x y}$ had a negative slope, indicating that $n$-type conduction and an ordinary Hall effect (OHE) were dominant at higher magnetic fields. It is well known that the anomalous part decreases, and the OHE becomes stronger with decreasing carrier concentration. It should be noted that $R_{0}$ is inversely proportional to the carrier concentration, which is illustrated in Fig. 4 for the $x=0.05$ film.

\section{Conclusion}

In conclusion, this study examined the magnetic and transport properties of $\mathrm{Cr}$ and $\mathrm{Sn}$ co-doped $\mathrm{In}_{2} \mathrm{O}_{3}$ films grown on $\mathrm{SiO}_{2} / \mathrm{Si}$ substrates by pulsed laser deposition. The results showed a clear enhancement of ferromagnetism and the appearance of an anomalous Hall effect as a result of electron-doping in Cr-doped $\operatorname{In}_{2} \mathrm{O}_{3}$. These results suggest that the high temperature ferromagnetism and transport properties of $\mathrm{Cr}$ and $\mathrm{Sn}$ codoped $\mathrm{In}_{2} \mathrm{O}_{3}$ films are reproducible. These features indicate that $\mathrm{Cr}$-doped $\mathrm{In}_{2} \mathrm{O}_{3}$ is a promising material for transparent oxide semiconductor spintronic devices operable at room temperature.

\section{Acknowledgements}

This study was supported by a Korean Council for University Education grant funded by the Korean Government (MOEHARD) for 2006 Domestic Faculty Exchange.

\section{References}

[1] W. Prellier, A. Fouchet, B. Mercey, J. Phys.: Condens. 
Matter 15, R1583 (2003).

[2] S. J. Pearton, C. R. Abernathy, M. E. Overberg, G. T. Thaler, D. P. Norton, N. Theodoropoulou, A. F. Hebard, Y. D. Park, F. Ren, J. Kim, L. A. Boatner, J. Appl. Phys. 93, 1 (2003).

[3] S. J. Pearton, W. H. Heo, M. Ivill, D. P. Norton, T. Steiner, Semicond. Sci. Technol. 19, R59 (2004).

[4] J. M. D. Coey, M. Venkatesan, C. B. Fitzgerald, Naure Mater. 4, 179 (2005).

[5] K. R. Kittilstved, N. S. Norberg, D. R. Gamelin, Phys. Rev. Lett. 94, 147209 (2005).

[6] J. Philip, A. Punnoose, B. I. Kim, K. M. Reddy, S. Layne, J. O. Holmes, B. Satpati, P. R. Leclair, T. S. Santos, J. S. Moodera, Nature Mater. 5, 298 (2006).

[7] C. Coutal, A. Azema, J.-C. Roustan, Thin Solid Films 288, 248 (1996).

[8] J. Philip, N. Theodoropoulou, J. S. Moodera, B. Satpati,
Appl. Phys. Lett. 85, 777 (2004).

[9] J. He, S. Xu, Y. K. Yoo, Q. Xue, H.-C. Lee, S. Cheng, X.-D. Xiang, G. F. Dionne, I. Takeuchi, Appl. Phys. Lett. 86, 052503 (2005).

[10] PDF card, PDF2 No. 71-2195, MDI JADE5 program (2003).

[11] H. J. Kim, J. H. Sim, H. Kim, S.-K. Hong, D. Kim, Y. E. Ihm, W. K. Choo, J. Magnetics 10, 95 (2005).

[12] S. N. Kale, S. B. Ogale, S. R. Shinde, N. Sahasrabuddhe, V. N. Kulkarni, R. L. Greene, T. Venkatesan, Appl. Phys. Lett. 82, 2100 (2003).

[13] K. H. Kim, K. J. Lee, D. J. Kim, H. J. Kim, Y. E. Ihm, C. G. Kim, S. H. Yoo, C. S. Kim, Appl. Phys. Lett. 82, 4755 (2003).

[14] C. L. Chien, C. R. Westgate, The Hall Effect and Its Applications (Plenum, New York, 1980). 